\title{
Research and Development for X-ray Optics and Diagnostics on the Linac Coherent Light Source (LCLS)
}

A. Wootton, J. Arthur, T. Barbee, R. Bionta, A. Jankowski, $R$. London, D. Ryutov, $R$. Shepherd, V. Shlyaptsev, $R$. Tachyn, A. Toor

This article was submitted to The $23^{\text {rd }}$ International Free Electron Laser Conference and the $8^{\text {th }}$ Free Electron Laser Users Group Darmstadt, Germany

August 20-24, 2001

\section{August 14, 2001}




\section{DISCLAIMER}

This document was prepared as an account of work sponsored by an agency of the United States Government. Neither the United States Government nor the University of California nor any of their employees, makes any warranty, express or implied, or assumes any legal liability or responsibility for the accuracy, completeness, or usefulness of any information, apparatus, product, or process disclosed, or represents that its use would not infringe privately owned rights. Reference herein to any specific commercial product, process, or service by trade name, trademark, manufacturer, or otherwise, does not necessarily constitute or imply its endorsement, recommendation, or favoring by the United States Government or the University of California. The views and opinions of authors expressed herein do not necessarily state or reflect those of the United States Government or the University of California, and shall not be used for advertising or product endorsement purposes.

This is a preprint of a paper intended for publication in a journal or proceedings. Since changes may be made before publication, this preprint is made available with the understanding that it will not be cited or reproduced without the permission of the author.

This report has been reproduced

directly from the best available copy.

Available to DOE and DOE contractors from the Office of Scientific and Technical Information

P.O. Box 62, Oak Ridge, TN 37831

Prices available from (423) 576-8401 http://apollo.osti.gov/bridge/

Available to the public from the National Technical Information Service

U.S. Department of Commerce 5285 Port Royal Rd., Springfield, VA 22161

http://www.ntis.gov/

OR

Lawrence Livermore National Laboratory

Technical Information Department's Digital Library

http://www.llnl.gov/tid/Library.html 


\title{
Research and Development for X-ray Optics and Diagnostics on the Linac Coherent Light Source (LCLS)
}

\author{
A. Wootton ${ }^{1}$, J. Arthur ${ }^{\mathrm{a}}$, T. Barbee, R. Bionta, A. Jankowski, R. London, D. Ryutov, \\ R. Shepherd, V. Shlyaptsev, R. Tatchyn ${ }^{\mathrm{a}}$, A. Toor,

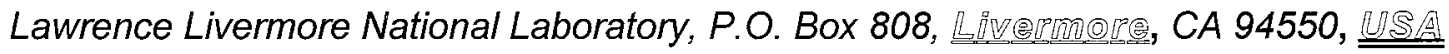

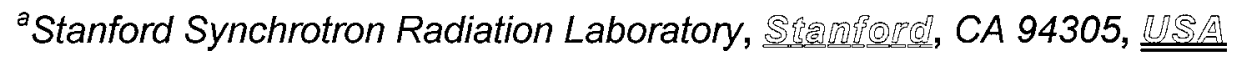

\begin{abstract}
The Linac Coherent Light Source (LCLS) is a 1.5 to $15 \AA$-wavelength free-electron laser (FEL), currently proposed for the Stanford Linear Accelerator Center (SLAC). The photon output consists of high brightness, transversely coherent pulses with duration $<300 \mathrm{fs}$, together with a broad spontaneous spectrum with total power comparable to the coherent output. The output fluence, and pulse duration, pose special challenges for optical component and diagnostic designs. We first discuss the specific requirements for the initial scientific experiments, and our proposed solutions. We then describe the supporting research and development program that includes: experimental and theoretical material damage studies; high resolution multilayer design, fabrication, and testing; replicated closed-form optics design and manufacturing; $\mathrm{BeB}$ manufacturing; and low-z Fresnel lens design, fabrication and testing. Finally some novel concepts for optical components are presented.
\end{abstract}

PACS: 41.50.+h Keywords: $x$-ray optics, LCLS, fourth generation light source

\section{Introduction}

The DOE Office of Basic Energy Science (OBES) has endorsed research and development (R\&D) towards a fourthgeneration light source [1] based on a LINAC-driven X-ray free electron laser (XFEL) [2]. Critical Decision 0, a statement of mission need, was signed on 13 June 2001. Such an XFEL would operate in an entirely new physics regime, self-amplified spontaneous emission (SASE), which has been now made possible by demonstrated advances in producing low-emittance electron beams (for a list of SASE-related experimental results, see http://www-ssrl.slac.stanford.edu/lcls/). The OBES is now funding R\&D to develop a specific proposal, the LINAC Coherent Light Source (LCLS) [3,4], which would utilize the last third of the SLAC LINAC and a precision undulator to produce $\mathrm{keV} x$-rays with unprecedented brilliance.

An x-ray optics working group has been created, whose responsibilities include:

Modeling the radiation field, and its interaction with matter,

Designing, testing and evaluating optical components for manipulating the beam, and for the initial experiments, Designing, testing and evaluating diagnostics to measure basic FEL photon beam characteristics.

The unprecedented brightness of the source, and the short pulse length, highlight demanding requirements as well as innovative design opportunities for the optical and diagnostics systems. Figure 1 illustrates that the normal fluence $\left(\mathrm{J} . \mathrm{cm}^{-2}\right)$

\footnotetext{
${ }^{1}$ Alan Wootton, Lawrence Livermore National Laboratory, PO Box 808, Livermore, CA 94550, phone (925) 422 6533, e-mail: woottonl@llnl.gov
} 
at the undulator exit far exceeds that required to melt materials. The challenge is somewhat alleviated downstream, where the natural divergence of the beam ( $1 \mu \mathrm{rad}$ at $8 \mathrm{keV}, 10 \mu \mathrm{rad}$ at $1 \mathrm{keV})$ reduces the fluence.

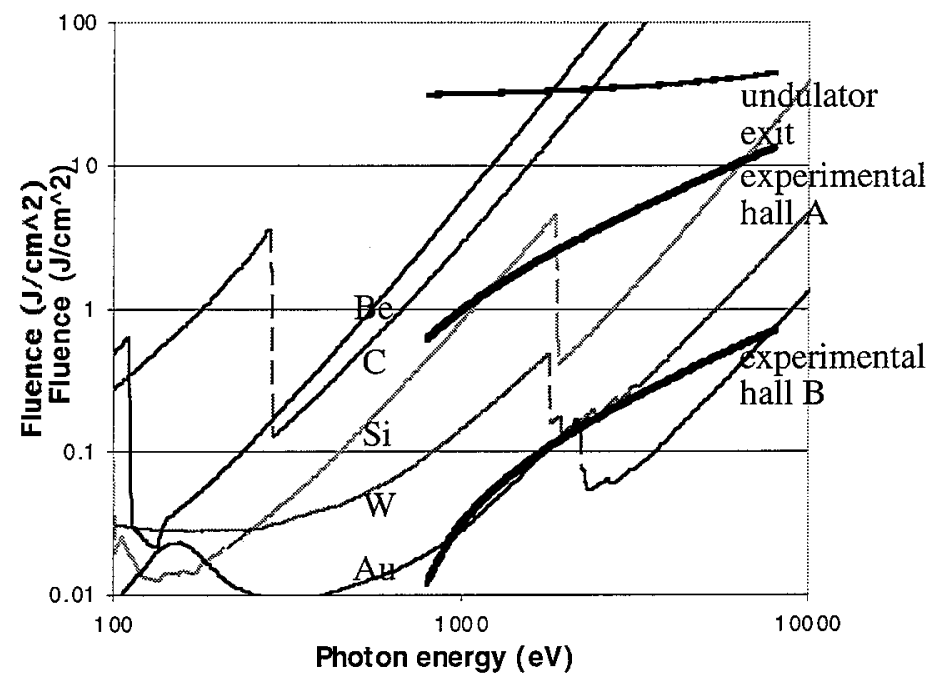

Figure 1. Normal incidence fluences from the LCLS coherent radiation beam, together with fluences required to bring various materials to their melting temperature $(\mathrm{Be}, \mathrm{C}, \mathrm{Si}, \mathrm{W}$, and $\mathrm{Au}$ ). The phase change energy is not included. Curves are shown for the LCLS coherent beam at the undulator exit, and at positions representative of the two experimental halls.

\section{The initial experimental requirements}

The optical and diagnostic requirements for the initial experiments are deduced from the published experimental campaign plans [5]. Some examples are found in Table 1. The challenges are not only dealing with the high fluence, but also beam manipulation and diagnosis.

\begin{tabular}{|l|l|l|l|}
\hline \multicolumn{1}{|c|}{ Campaign } & Energy (keV) & \multicolumn{1}{|c|}{ Challenging Requirements } & \multicolumn{1}{c|}{ Fluence $\left(\mathbf{J}^{-c^{-2}}\right)$} \\
\hline Atomic physics & $\sim 1$ & Remove SR and third harmonic, focus to $<0.1 \mu \mathrm{m}$ & 0.54 \\
\hline Plasma physics & $\sim 8$ & $\begin{array}{l}\text { Beam focus to }<10 \mu \mathrm{m}, \text { synchronize to a short pulse } \\
\text { laser }\end{array}$ & 8.45 \\
\hline $\begin{array}{l}\text { Nanoscale } \\
\text { dynamics }\end{array}$ & Up to 24 & Beam split with inter-beam delays from $10^{-12}$ to $10^{3} \mathrm{~s}$ & 0.614 at $8 \mathrm{keV}$ \\
\hline Biology & 0.8 to 8 & $\begin{array}{l}\text { Beam temporal compression or time slicing, and } \\
\text { focusing, to } 10^{8} \text { photons/A }{ }^{2} \text {; synchronization to target. }\end{array}$ & 0.01 to 0.54 \\
\hline Diagnostics & 0.8 to 24 & Pulse temporal history, synchronization, coherence & 0.008 to 0.51 at $8 \mathrm{keV}$ \\
\hline
\end{tabular}

Table 1. Some of the optical requirements for a subset of the initial LCLS science experiments.

To deal with the fluence, four specific hardware solutions are proposed:

1. A far field experimental hall to reduce energy densities by natural divergence,

2. A gas absorption cell to continuously attenuate by $>10^{4}$, 
3. Low- $Z$ optics that are damaged least (e.g. a phase zone plate for warm dense matter experiments),

4. Grazing incidence optics that increase the optical footprint and reflect most incident power.

In most cases the experiments that require the most challenging beam manipulation, including time compression, time slicing, beam splitting and beam delay, are planned for the far hall. Should these hardware solutions be insufficient, two other categories are being explored. First, non-solid state (i.e. liquid and plasma) optics that are readily replenished have been studied. Second, there is the option of dynamic solid-state optics, that are spatially translated between shots.

\section{Supporting research and development}

\subsection{Material damage studies}

Energy fow for $x$-ray interaction with low $Z$ solids

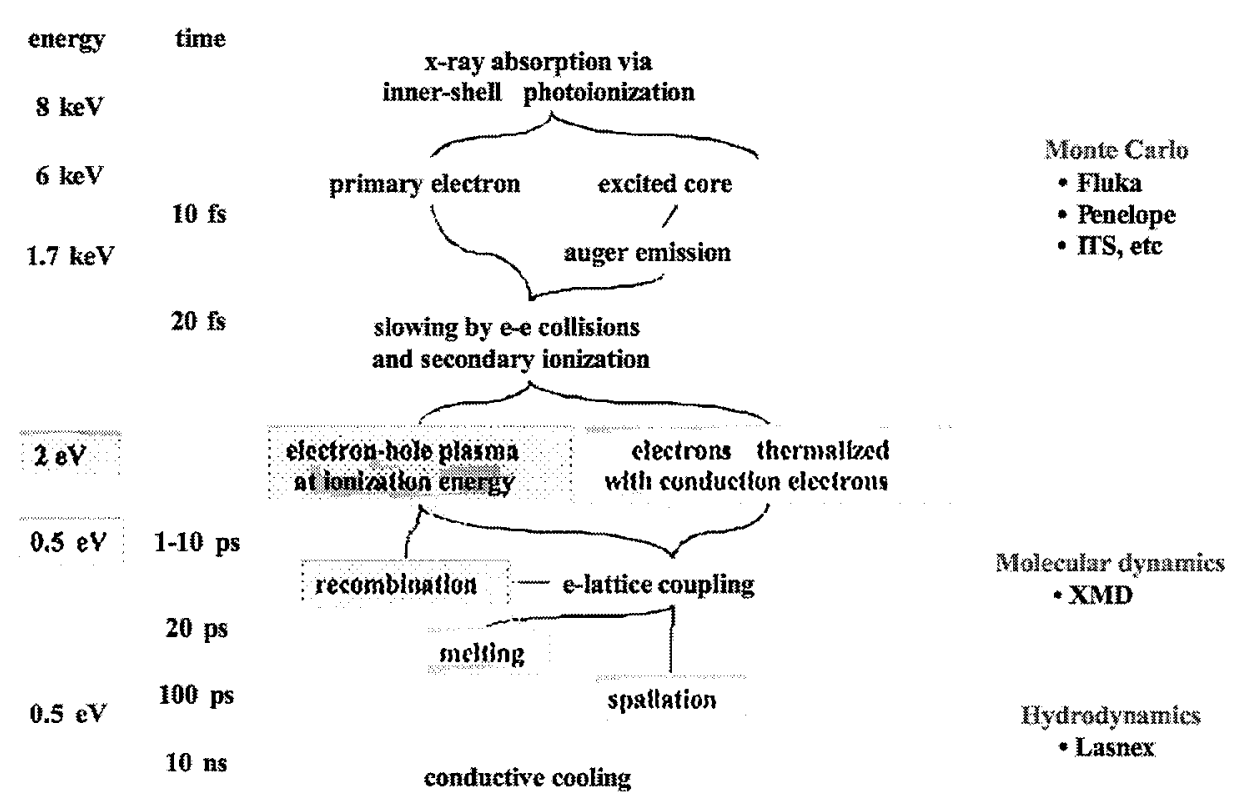

Figure 2. Processes involved in x-ray absorption.

Figure 2 illustrates the expected energy deposition processes for $\mathrm{x}$-rays interacting with a low-Z solid. Processes, typical time scales and typical energies (first $\mathrm{x}$-ray, then electron, finally electron and ion), are shown for an $8 \mathrm{keV}$ photon interacting with $\mathrm{Si}$, one of the primary materials being considered for mirrors and diffraction crystals. The absorption of $\mathrm{x}$-rays proceeds primarily by inner-shell photo-ionization events, which occur essentially instantaneously. This is followed by the emission of a primary photoelectron of energy about $6 \mathrm{keV}$ and the creation of a core-excited atom. The atom relaxes mainly by the auger process in about $10 \mathrm{fs}$ emitting an auger electron of energy $1.7 \mathrm{keV}$. Another auger process will lead to a lower energy electron and the creation of an atom with a valence-shell hole. The photo-and auger electrons then slow down by secondary ionization processes and by electron-electron (elastic) collisions. The slowing down is estimated to occur in about $20 \mathrm{fs}$, dominated by the highest energy electrons. This leads to an intermediate state of the matter with warm electrons ( 0.5 to $2 \mathrm{eV}$, at doses near the damage threshold). The actual temperature depends on the type of material; in metals, the energetic electrons will likely thermalize with the existing conduction band electrons, leading to a relatively low electron temperature, while in semi-conductors and insulators, the electrons will slow until they drop to the band-gap energy, at which they can no longer create new electrons. This energy is $2 \mathrm{eV}$ in silicon. The thermalized electrons will then interact with the atoms and ions in the lattice on a much longer timescale. Recombination and electron-lattice coupling take place in 1-10 ps. Once the electrons and ions have equilibrated, bulk effects commence. The chief effects of interest are melting (timescale 
$\sim 20 \mathrm{ps}$ ), and the propagation of stress pulses (timescale $\sim 100 \mathrm{ps}$ ), which may lead to spallation or fracture. Cooling of the $\mathrm{x}$-ray heated region occurs on a still longer timescale of about $10 \mathrm{~ns}$, for a $1 \mu \mathrm{m}$ deep deposition region, characteristic of grazing incidence $x$-rays. Thus damage is expected only after $>20 \mathrm{ps}$, well after the FEL pulse has gone, and a working assumption for precluding material damage that would affect $\mathrm{x}$-ray properties is:

'There will be no changes to material $\mathrm{x}$-ray properties during the $\mathrm{x}$-ray pulse, and no irreversible changes at any time, as long as the fluence is kept $<$ about $1 / 2$ that required to bring the material temperature to melt'.

This assumption is still being explored, both theoretically and experimentally. In Figure 2 relevant simulation codes are shown in the respective energy or time range; Monte Carlo calculations of photoelectron transport and associated energy deposition profiles are under way, as are hydrodynamic modeling calculations of spallation and repetitive thermal stress fracture. Experimentally, data from preliminary experiments using a sub-ps, $800 \mathrm{~nm}$ laser beam to damage a Si wafer are consistent with the working assumption above. A more complete understanding will come from planned experiments using sub-ps x-ray pulses to damage, and measuring changes to material properties in real time. Three sources are being considered: a) the Comet $x$-ray laser at $85 \mathrm{eV}$ [6], or laser-produced Kalpha photons, which are either b) focused to the sample, or c) placed in proximity $(<100 \mu \mathrm{m})$ to the sample.

\subsection{High resolution multilayers}

A series of multilayer optics, suitable for time slicing, have been designed, built and tested. The conclusions are:

The SSRL beamline can be used as a high precision calibration facility for multilayer mirrors $\mathrm{B}_{4} \mathrm{C}-\mathrm{C}$ multilayers are restricted in applicability by their half life at room temperature, $\sim 1$ month $\mathrm{WSi}_{2} \mathrm{Si}$ and $\mathrm{MSi}_{2} \mathrm{~S}$ multilayer mirrors worked almost as well as predicted at $8 \mathrm{keV}$. One $\mathrm{MSi}_{2} \mathrm{Si}$ had an energy resolution of $\mathrm{E} / \Delta \mathrm{E}=292$, and a reflectivity $55<\mathrm{R}<67$. Thus we expect to be able to produce mirrors suitable for both wavelength filtering and time slicing the LCLS beam (requiring $300<\mathrm{E} / \Delta \mathrm{E}<1000$ ).

\subsection{Replicated optics}

Focusing for the atomic physics experiments may be achieved using a parabaloid mirror. Two techniques are considered for manufacture. First, only that part of the parabaloid that will reflect the photons can be numerically machined, i.e. a section $\sim 7 \mathrm{~cm}$ long by $\sim 2 \mathrm{~mm}$ wide. Alternatively full parabaloids can be made by sputter depositing layers of materials (e.g. Be, or C) onto a super-smooth mandrel, thus forming an optic from the inside outward. During mandrel removal, the stress-relieved specular (and if required multilayer) reflecting surfaces and supporting electroplated shells do not deform, and consequently produce super-smooth surfaces comparable to the initial mandrel surface (i.e. rms $\approx 3$ to $4.5 \AA$ ) with figures replicating the mandrel figure. It may also be possible to form a Si optic using mechanical-chemical polishing. Stress-relieved specular (and if required multilayer) reflecting surfaces and supporting electroplated shells do not deform. Typical specular reflecting material structures are between $25 \mathrm{~nm}$ and $50 \mathrm{~nm}$ thick.

\subsection{BeB compounds}

A suitable material for high fluence optics is $\mathrm{Be}$, because of its low photo-absorption cross-section and high melting point. Unfortunately, pure Be (devoid of impurities as oxygen which is found in the powder processed materials) is a brittle material and susceptible to machining damage that can lead to degraded properties particularly under high strain rate conditions [7]. One possibility to overcome this limitation is to refine the grain size in order to prepare a more homogeneous and isotropic material for processing to a high surface finish. The synthesis of Be-B compounds as vapor deposited coatings have shown 
great promise in reducing grain size to the nano scale [8]. For bulk applications of interest, two Be-B compounds (with 3\% and $10 \% \mathrm{~B}$ ) have been vacuum cast and slow cooled for application consideration as mirrors. Although the grain size is reduced by more than a factor of three for the $\mathrm{Be}-3 \% \mathrm{~B}$ sample in comparison to the $100 \mu \mathrm{m}$ grain size for the cast pure $\mathrm{Be}$ as shown in the optical micrographs of Figure 3, the grain size remains larger than desired for low rms roughness surfaces. A possible path to further reduce the grain size and surface roughness is to increase the quench rate of the vacuum casting.
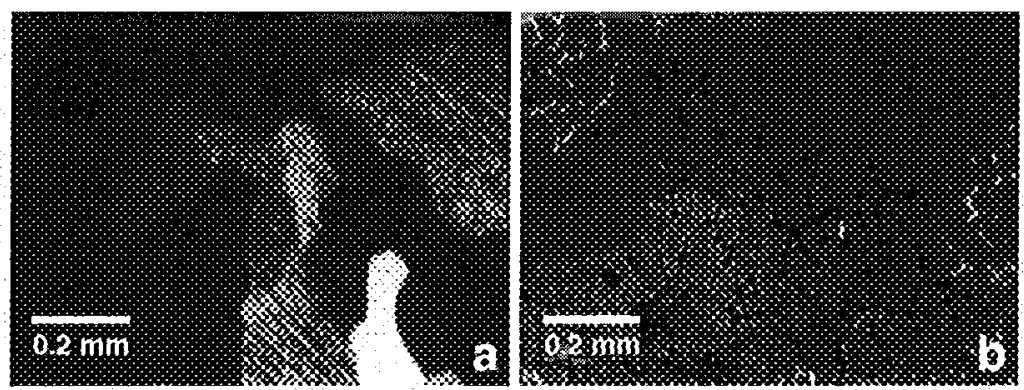

Figure 3. Vacuum cast samples of (a) pure $\mathrm{Be}$ and (b) $\mathrm{Be}-3 \% \mathrm{~B}$ are imaged using polarized light after being prepared in cross-section by mechanically grinding using $\mathrm{SiC}$ then rough-polished using a colloidal suspension of diamond particles.

\subsection{Low-z phase zone plates}

A blazed carbon Fresnel zone-plate (a Fresnel lens) is chosen for the preliminary plasma physics experiments. A radiograph is shown in Figure 4 of an aluminum version that has been successfully tested. The lens is carved into the face of a $\mathrm{C}$ disk and mounted over a hole drilled through a $\mathrm{Cu}$ mount. The $\mathrm{C}$ disk is $650 \mu \mathrm{m}$ thick except in the center, where it thins down to $400 \mu \mathrm{m}$. The active portion of the lens is $200 \mu \mathrm{m}$ in diameter and consists of concentric grooves machined to a maximum depth of $18.8 \mu \mathrm{m}$.

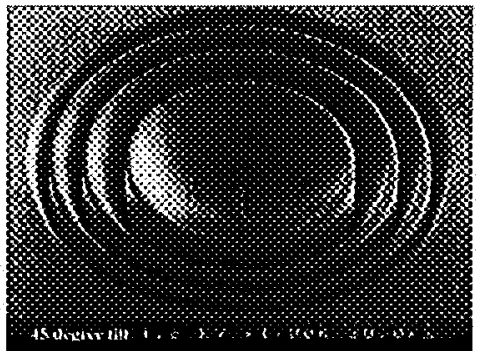

Figure 4. A radiograph of an $\mathrm{Al}$ Fresnel lens, with feature size $\sim 10 \mu \mathrm{m}$, groove depth $\sim 20 \mu \mathrm{m}$.

\subsection{Novel concepts}

\subsubsection{Plasma optics}

Capillary discharges produce plasmas with density profiles suitable for focusing. The capillary material supplies the plasma, which is Joule heated in the center and cooled at the edge. Pressure balance provides the inverted density profile. A design study for such a transmissive system based on $\mathrm{LiH}$ as been completed, and shows it could work in the 3 to $10 \mathrm{~A}$ region with the full fluence, with focal spot sizes $<1 \mu \mathrm{m}$, lasting for $>10^{3}$ shots [9].

\subsubsection{Liquid optics}

A new class of high-flux renewable liquid optics is proposed, with size from a fraction of a $\mathrm{cm}$ to a few $\mathrm{cm}[10]$. Working fluid is pressed by a piston through a porous substrate (e.g. fused capillaries) to form a film, $\sim 10$ to $100 \mu \mathrm{m}$ thick. After the FEL pulse, the film is sucked back through the substrate by a reversed motion of the piston, and formed anew before the next 
pulse. The working surface of the film is maintained parallel to the substrate by capillary forces. A properly made film can be arbitrarily oriented with respect to the gravitational force, and controlled by electrostatic and magnetic forces, allowing one to make parabolic mirrors and reflecting diffraction gratings.

\subsubsection{Thermo-elastically controlled optics}

Consider a polished flat metal surface that has a sufficient reflectivity for $\mathrm{x}$-rays at a grazing incidence. This is then irradiated by a subsidiary optical or UV laser pulse, at an angle near normal, and with wavelength corresponding to as strong as possible surface absorption. The subsidiary laser heats the metal surface in a desired surface pattern, and thermal expansion creates a desired surface relief - a focusing or defocusing mirror. If after the passage of the main FEL pulse the surface is damaged, the mirror plate can be translated. A preliminary design study shows that a $10 \mathrm{~W}, 10 \mathrm{~mJ}$ laser could produce a focusing mirror on an Al plate

\section{Summary and conclusions}

The Linac Coherent Light Source is a proposal for the first in a new class of light sources. The unprecedented brightness implies a high energy loading on optics and diagnostics, and offers the opportunity for design innovations. The R\&D program includes developing high resolution multilayers, low $Z$ Fresnel lenses, replicated optics and low $Z$ materials in general. Optics whose surfaces can be continuously replenished are also being considered (e.g. liquids and plasmas). Theoretical and experimental studies of photon-material interactions are being pursued, to understand both long time (thermal diffusion) and short time (sub ps) phenomena.

\section{Acknowledgements}

This work was performed under the auspices of the U.S. Department of Energy by the University of California, Lawrence Livermore National Laboratory under contract No. W-7405-ENG-48.

\section{References}

1. Report of the Basic Energy Sciences Advisory Committee, Panel on Novel Coherent Light Sources, January 1999, available at: http://www.er.doe.gov/production/bes/BESAC/NCLS_rep.PDF.

2. J. B. Murphy and C. Pellegrini, "Introduction to the Physics of the Free Electron Laser," in Frontiers of Particle Beams, M. Month, S. Turner, eds, Lecture Notes in Physics No. 296, H. Araki et al, eds., Springer-Verlag, Berlin, pp. 163212 (1988).

3. M. Cornacchia et al., "Performance and design concepts of a free-electron laser operating in the x-ray region," SPIE Proceedings 2988, p2, (1997).

4. J. Arthur et al., LCLS Design Study Report, Internal Stanford University report SLAC-R-521, Rev. December 1998 UC414 (1998).

5. LCLS, The First Experiments, available at: http://www-ssrl.slac.stanford.edu/lcls/papers/LCLS experiments 2.pdf.

6. Y. Li, J. Dunn, J. Nilsen, A. Osterheld and V. Shlyaptsev, "Recent Progress of plasma X-ray lasers at LLNL", Proc. Free Electron Laser Conference 2000, Durham, NC, Aug 13-18, 2000 (200), and LLNL report, UCRL-JC-129260-ABS (2000).

7. D. Gallagher and R. Hardesty, "Machining of Beryllium" in Metals Handbook Vol 16 - Machining (ASM International, Metals Park) pp. 870-3 (1989).

8. A. Jankowski, et al., "Nano-crystalline and hard boron-alloyed Be coatings," Mater. Res. Soc. Symp. Proc. Vol. 593 pp. 489-492 (2000).

9. V. N. Shlyaptsev and A. Toor, "Plasma lens for high-flux x-ray radiation", Proceedings of the 46 annual SPIE meeting, San Diego, CA. paper 4500-15 (2001).

10. D. Ryutov and A. Toor, "Renewable liquid optics with magneto-electrostatic control ", Proceedings of the 46 annual SPIE meeting, San Diego, CA. paper 4500-14 (2001). 


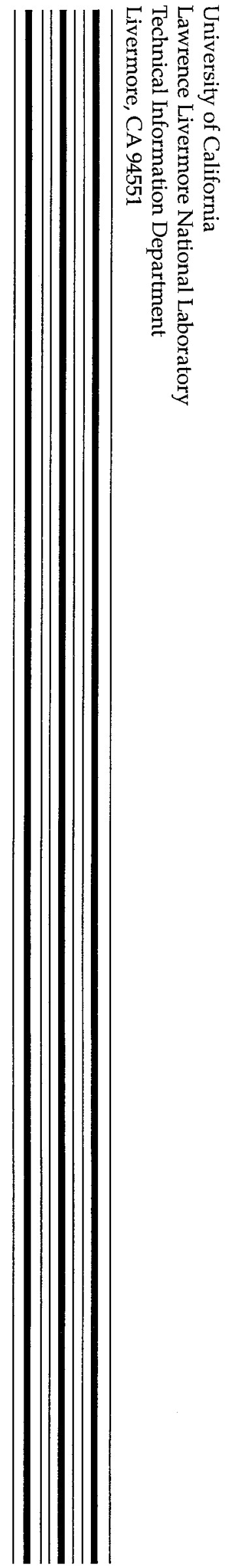

